\title{
Incorporating inertia into multiagent systems
}

\author{
W. C. Man and H. F. Chau* \\ Department of Physics, University of Hong Kong, Pokfulam Road, Hong Kong \\ and Center of Theoretical and Computational Physics, University of Hong Kong, Pokfulam Road, Hong Kong
}

(Received 10 October 2005; published 3 March 2006)

\begin{abstract}
We consider a model that demonstrates the crucial role of inertia and stickiness in multiagent systems, based on the minority game. The inertia of an agent is introduced into the game model by allowing agents to apply hypothesis testing when choosing their best strategies, thereby reducing their reactivity toward changes in the environment. We find by extensive numerical simulations that our game shows a remarkable improvement of global cooperation throughout the whole phase space. In other words, the maladaptation behavior due to over-reaction of agents is removed. These agents are also shown to be advantageous over the standard ones, which are sometimes too sensitive to attain a fair success rate. We also calculate analytically the minimum amount of inertia needed to achieve the above improvement. Our calculation is consistent with the numerical simulation results. Finally, we review some related works in the field that show similar behaviors and compare them to our work.
\end{abstract}

DOI: 10.1103/PhysRevE.73.036106

PACS number(s): 89.65.Gh, 02.50.-r, 05.40.-a, 89.75.-k

\section{INTRODUCTION}

There is a growing interest in studying artificial agentinteracting models which are able to generate global behaviors found in social, biological, and economical systems [1]. Examples such as matching games [2] and ideal gas models of trading markets [3] show that this approach commonly used by physicists can be nicely applied to problems lying outside the discipline. One exciting fact is that these artificial models, although they contain simple governing rules, can still generate nontrivial global cooperative behaviors $[4,5]$. In these self-organized complex systems, agents can reach equilibrium states through adaptation, a dynamical learning process initiated by the feedback mechanism present in these systems.

People possesses inertia when making decisions and switching strategies in economical systems. Conceptually, this inertia is similar to the one used by Newton to describe body motions in the physical world. It refers to how reluctant a person is to drop his/her current economics plan and look for another one, just as an object is reluctant to change its motion state. Inertia may originate from (1) the cost needed to change strategies, (2) the low sensitivity toward a change in environment, and (3) the loss-aversion behavior in humans [6]_-people love to fight back from loss [7]. As different bodies may have different mass in classical physical systems, different people may carry different inertia in economical markets. In this paper, we introduce a simple model to study the idea of inertia. This model gives a striking improvement of cooperative behavior, such as removal of maladaptation [8] and dynamical increase of diversity among agents, without any necessity to alter the initial conditions and payoff mechanism. Actually, studies of a few variants of the minority game (MG) also show improvement in cooperation [9-13]. We shall further discuss their results and compare

\footnotetext{
*Corresponding author: hfchau@hkusua.hku.hk
}

with ours in Sec. VI after finishing reporting our model and results.

Our model is a modification of a famous econophysical model known as the MG, proposed by Challet and Zhang in $1997[5,14]$. The MG is a simple game model that captures the minority seeking behavior found in stock markets and resource competition. (See Refs. [15-17] for an overview of econophysics and the MG.) In the MG, $N$ agents struggle to choose between two options repetitively, either buying (0) or selling (1) in each turn. Those who have chosen the minority side are winners at that turn and are awarded $\$ 1$, otherwise they lose $\$ 1$. The only information they receive is the history of the game, which is a binary bit string composed of the minority choices of the previous $M$ turns. A strategy is a map from the set of all possible histories to the set of two options. If a strategy predicts the minority correctly, it gains 1 virtual score point, otherwise it loses 1 virtual score point. Each agent is assigned $S$ strategies once and for all at the beginning of the game in order to aid his/her decisions. In the standard MG, an agent makes decisions based on his/her best current strategy at hand, namely, the one with the highest virtual score.

Clearly, there are $2^{M}$ possible histories and hence $2^{2^{M}}$ available strategies. However, out of the whole strategy space, only $2^{M+1}$ of them are significantly different. The diversity of the population is measured by $\alpha$, which is equal to $2^{M+1} / N S$. The smaller $\alpha$, the more similar are the strategies held by agents. Up to a first-order approximation, the dynamics of the MG is determined by this control parameter $\alpha$ [18-20].

The most obvious macroscopic observable in the $\mathrm{MG}$ is perhaps the variance of optional attendance per agent, $\sigma^{2} / N$. It represents the wastage of the system and fluctuation of resource allocation; the smaller $\sigma^{2} / N$, the more the whole population benefits. Researchers found that $\sigma^{2} / N$ falls below the value it would have if all agents make their choices randomly in a certain range of $\alpha$. This indicates that agents are cooperating although they are independent and selfish. More 
importantly, there is a phase transition at the critical point $\alpha_{c}$ which divides the $\sigma^{2} / N$ against $\alpha$ curve into the so-called symmetric phase $\left(\alpha<\alpha_{c}\right)$ and asymmetric phase $\left(\alpha>\alpha_{c}\right)$ [21].

\section{OUR MODEL}

To incorporate inertia into the $\mathrm{MG}$, we introduce a modification - the hypothesis testing minority game (HMG). Hypothesis testing is a standard statistical tool to test whether an effect emerging from an independent variable appears by chance or luck. In the standard version of the MG, the best strategy is defined as the strategy with the highest virtual score. In the HMG, however, an agent $k$ determines his/her own best strategies by testing the following null hypothesis $H_{0}$ : The current strategy $\mathcal{S}_{k, 0}$ performs better than the other strategy $\mathcal{S}_{k, 1}$ available to agent $k$. Note that we have restricted ourselves to the simplest case $S=2$, but the model can be easily extended to $S>2$ cases under the same formalism. This agent possesses a sustain level $I_{k} \geqslant 1 / 2$ on his/her current strategy $\mathcal{S}_{k, 0}$, which is the same as the confidence level on the validity of the null hypothesis we commonly use in hypothesis testing (that is, the acceptance area of a standard normal). This $I_{k}$ defines how much he/she could sustain the underperformance of $\mathcal{S}_{k, 0}$ and thereby represents his/her inertia.

The $H_{0}$ of a particular agent $k$ can be quantitatively written as

$$
H_{0}: \frac{\Omega_{k, 0}\left(\tau_{k}\right)-\Omega_{k, 1}\left(\tau_{k}\right)}{\delta_{k}}>x_{k},
$$

where

$$
\frac{1}{\sqrt{2 \pi}} \int_{x_{k}}^{+\infty} e^{-x^{2} / 2} d x=I_{k} .
$$

Here, $\Omega_{k, j}\left(\tau_{k}\right)$ is the virtual score of a particular strategy $\mathcal{S}_{k, j}$ at $\tau_{k}$, where $\tau_{k}$ is the number of time steps counted from his/her adoption of $\mathcal{S}_{k, 0}$ for that individual agent. The dominator $\delta_{k}$ represents the fluctuation of the strategies' performance perceived by the agent. An agent $k$ would continue to stick on his/her current strategy $\mathcal{S}_{k, 0}$ until $\Omega_{k, j}\left(\tau_{k}\right)$ descends outside his/her sustain level. Then he/she has to admit that $H_{0}$ is not likely to be true, rejects it, and shifts to the other strategy. After a change of strategy, the virtual scores of both strategies are reset to 0 and $\tau_{k}$ is set back to 1 .

The higher the value of $I_{k}$, the milder his/her response and the more reluctant he/she will be to shift strategies. In this way, $I_{k}$ plays the role of the inertia of an agent in this game. Agents with $I_{k}=1 / 2$ would be most similar to the standard MG agents, employing the strategy with the highest virtual score. However, there are still two differences: these HMG agents will still stick on the current strategy in the case of a tie in virtual scores, and the virtual scores will be reset after shifts in strategies.

We remark that randomness is involved in only three places in the HMG, namely, (1) the initial assignment of strategies and inertia; (2) the choice of a new strategy in case of a tie in the virtual scores of the alternative strategies when a player has decided to drop the current one; as well as (3) the determination of the winning side in the case of a tie. Thus, the dynamics of the HMG is deterministic when played by an odd number of agents each carrying two strategies.

\section{PURE POPULATION WITH RANDOM WALK APPROXIMATION}

We have performed extensive numerical simulations on our model. With the presence of inertia among agents, every agent needs a longer time to make up his/her mind and the equilibration time in the HMG is lengthened. We take the value of $\sigma^{2}$ every 1000 time steps and regard the system as having equilibrated if the percentage difference of successive measurements is smaller than $\epsilon=10^{-6}$. Upon equilibration, we take our measurement by recording the dynamics of the next 25000 time steps. Furthermore, we repeat this data taking procedure 150 times, each with an independent set of initial conditions.

In a population where everyone tries to cling to the minority side as long as possible, agents may have different inertia $I_{k}$, and some may have no inertia at all (standard MG agents). We first study the behavior of the HMG when every agent has the same $I_{k}$. (We shall move on to study the mixed population case in later sections.) We begin our study by determining the value of $\delta_{k}$, a perception of agents of the fluctuation of the virtual score difference between two strategies. A naive guess would be to assume that $\Omega_{k, j}\left(\tau_{k}\right)$ performs a random walk for all strategies $j$ throughout the game; then $\delta_{k}$ equals $\sqrt{2 \tau_{k}}$.

Figure 1 shows a plot of the variance of attendance for a particular option $\sigma^{2} / N$ against the control parameter $\alpha$ for different inertia $I$, with $\delta_{k}$ set to $\sqrt{2 \tau_{k}}$. There is a huge drop of $\sigma^{2} / N$ when $I$ is sufficiently large, especially in the symmetric phase when $\alpha$ is small (see Fig. 2). Not just is the maladaptation in the symmetric phase greatly reduced, but the cooperation between agents is also improved in the asymmetric phase for certain values of $I$.

The reduction of system wastage in the asymmetric phase $\left(\alpha>\alpha_{c}\right)$ is believed to result from increasing stickiness of agents on current strategies and elongation of their observation time. This leads to an increase of frozen agents (see Fig. 3 ) and a more effective crowd-anticrowd cancellation, resulting in better cooperation [21-23].

However, things become more complicated when $\alpha<\alpha_{c}$. From now on, this paper will focus on the striking improvement of cooperation in the symmetric phase. The removal of maladaptation in this region is directly related to the disappearance of periodic dynamics that is normally present in the standard MG. The periodic dynamics is a result of oversampling of strategy space and common zero initial conditions among agents when $\alpha<\alpha_{c}$, accounting for the high volatility in the symmetric phase. It is reflected in a prominent period$2^{M+1}$ peak in the autocorrelation of the attendance time series of a particular option [8,19,20,24,25]. Figure 4 shows evidence of this postulate: as shown from the autocorrelation function, periodic dynamics appears in the case $I=0.53$ which has high $\sigma^{2} / N$ in Figs. 1 and 2, while the low $\sigma^{2} / N$ 


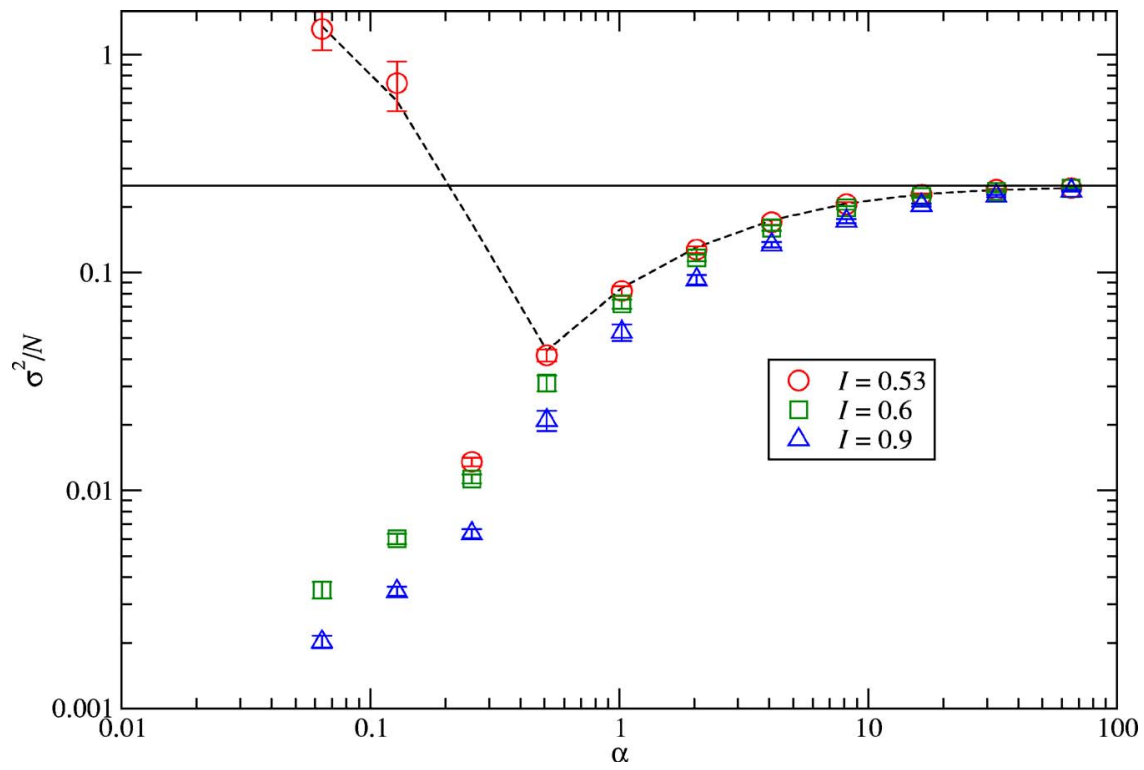

FIG. 1. (Color online) The variance of attendance per agent, $\sigma^{2} / N$, against the system complexity $\alpha$ for $I=$ (a) 0.53 , (b) 0.6 , and (c) 0.9 , setting $\delta_{k}=\sqrt{2 \tau_{k}}$. Here, $N=501$ and $S=2$. The dashed line represents the $\sigma^{2} / N$ curve in the standard MG.

cases $I=0.6$ and 0.9 show no trace of this signal.

What is the critical limit of $I$ in order to remove the maladaptation? To answer this, we have to look closely into the periodic dynamics that governs the maladaptation in the symmetric phase. An earlier study stated that virtual scores of strategies are likely to reset to 0 every $2^{M+1}$ number of steps through the periodic dynamics in the symmetric phase. Initially all strategies have score point 0 ; whenever a strategy $\beta$ wins a bet in a particular $\mu$, most agents would rush to $\beta$, which is 2 score points ahead of its anticorrelated partner $\bar{\beta}$ in the next appearance of $\mu$. It is likely that they would lose due to this overcrowding. In this manner, the virtual scores of all strategies are reset at this stage. This loop repeats with interval $2^{M+1}$ and leads to the large fluctuation of option attendance in the symmetric phase [26].

Therefore, the question becomes when this reset and oscillate mechanism will disappear. Actually, the periodic dynamics is destroyed when agents are no longer sensitive enough to immediately shift to a particular strategy after winning a bet. The criterion for this situation to occur is given by

$$
\frac{-2}{\sqrt{2\left(2^{M+1}\right)}}<x_{k},
$$

where $x_{k}$ satisfies Eq. (2). If the value of $I$ satisfies the inequality (3), agents will no longer be constrained by the periodic dynamics every $2^{M+1}$ steps. Then, a re-recognizing process will begin. In the standard MG, all identical strategies have the same virtual scores throughout the game. However, in the HMG agents clear all virtual scores after changing strategies. This move is done in various time steps for different agents, depending on the combination of strategies in their hands. Hence, the scores of identical strategies eventually diverge if they are held by different agents, and these strategies may be employed again at many times in the fu-

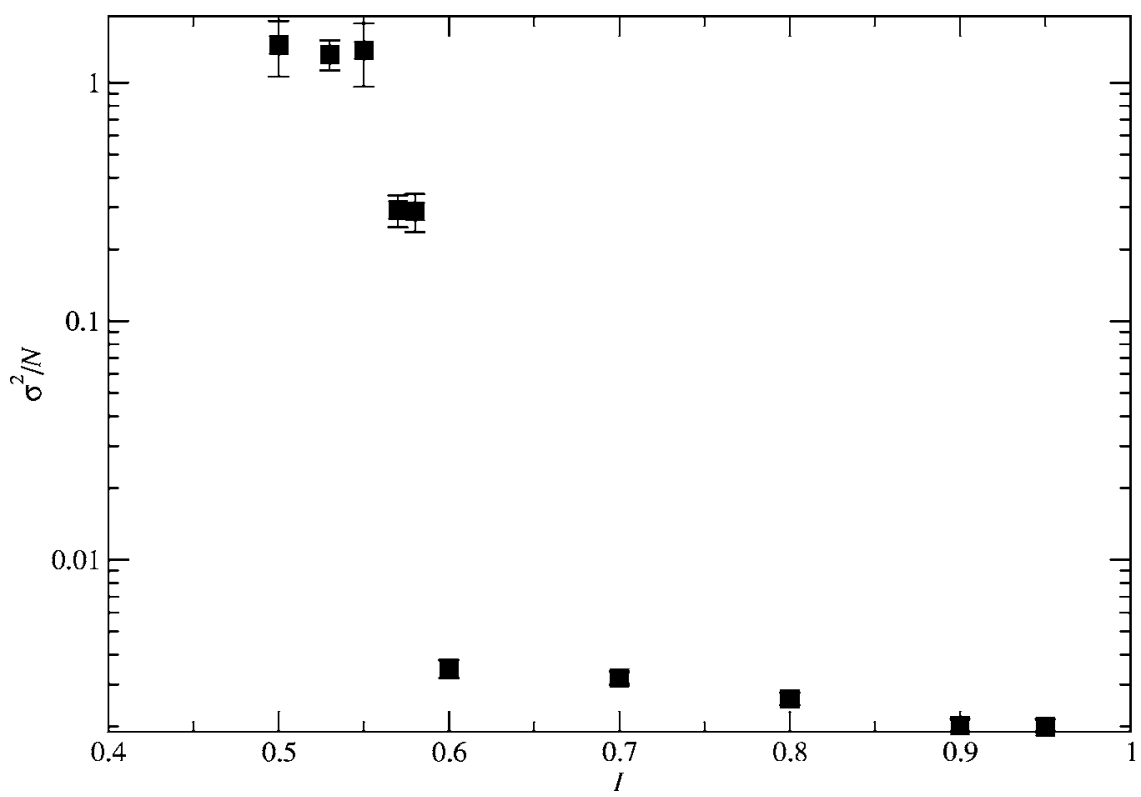

FIG. 2. The variance of attendance per agent, $\sigma^{2} / N$, against $I$ at $\alpha=0.06$, setting $\delta_{k}=\sqrt{2 \tau_{k}}$. Here, $N=501, S=2$, and $M=5$. 


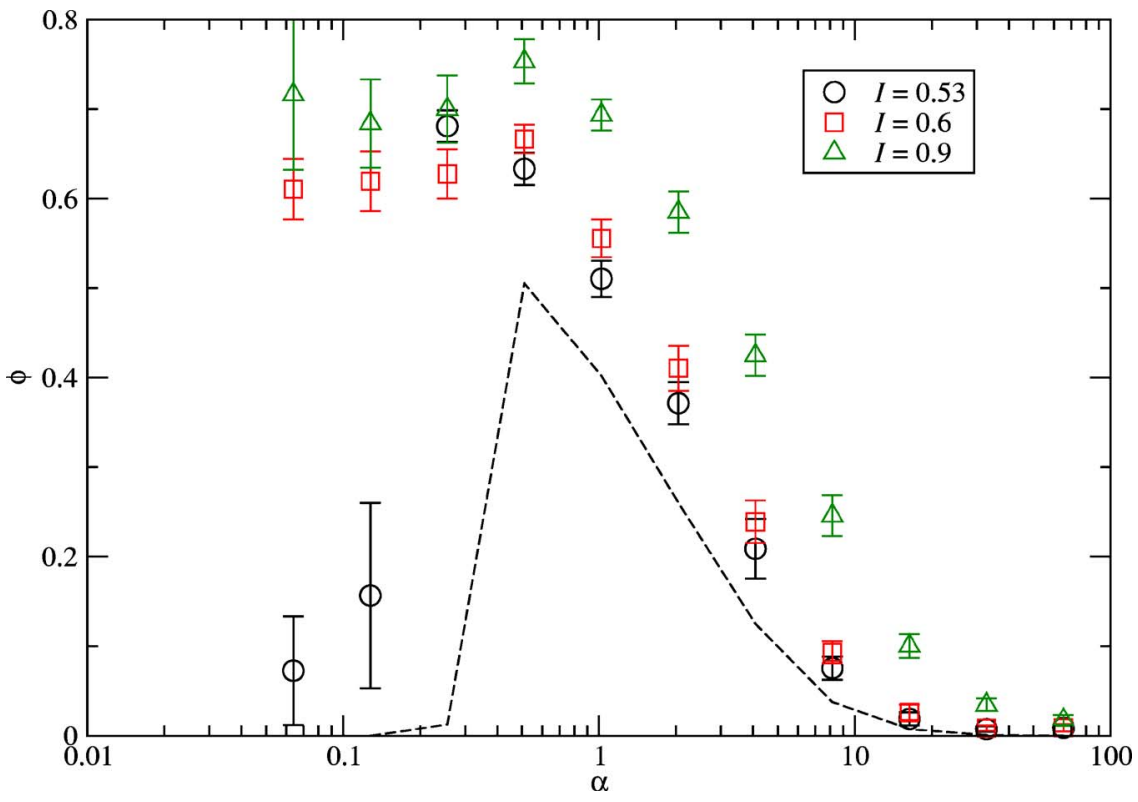

FIG. 3. (Color online) The frozen probability $\phi$ against $\alpha$ for different $I$ by setting $\delta_{k}=\sqrt{2 \tau_{k}}$. The dashed line represents the frozen probability of the standard agents in the MG. Here, $N=501$, $S=2$, and $M=5$. ture. The net effect of this re-recognizing process is to diversify strategies in the population intrinsically. In this way, both the oversampling and overcrowding found in the symmetric phase are relaxed, lowering the volatility.

For instance, when $M=5$, the limit $x_{c}$ equals $-2 / \sqrt{2\left(2^{5+1}\right)}=-0.177$; that is, $I \simeq 0.57$. This criterion is confirmed in Figs. 2 and 4-all cases that show no periodic dynamics, satisfy Eq. (3), and have low variance. Note that for the cases where I does not exceed this limit, their correlation signals are much stronger than that of the standard $\mathrm{MG}$ [see Fig. 4(b)]. It is because the dynamics of the HMG is more deterministic than that of the standard MG as HMG agents will continue to stick on the current strategy when facing a tie in strategy virtual scores, which happens during a reset. That means their actions repeat during this reset and the system path is more likely to repeat, resulting in stronger correlation. This is like removing the random dice in the standard MG when facing a tie in virtual scores; a periodic signal as in strong as this case is also obtained.

\section{PURE POPULATION WITH RUN TIME $\delta_{k}$}

Actually, the movement of the virtual score difference between two strategies is not likely to perform a random walk. Another possible way to perceive $\delta_{k}$ is to put it as the actual standard deviation of this difference in run time, which represents a more realistic market scenario. That is,

$$
\delta_{k}=\sqrt{\left\langle\left[\Omega_{k, 0}\left(\tau_{k}\right)-\Omega_{k, 1}\left(\tau_{k}\right)\right]^{2}\right\rangle_{\tau_{k}}-\left\langle\Omega_{k, 0}\left(\tau_{k}\right)-\Omega_{k, 1}\left(\tau_{k}\right)\right\rangle_{\tau_{k}}^{2}} .
$$

The results are very similar to the previous case, and are shown in Figs. 5-7. However, the critical value of $I$ for the (a)

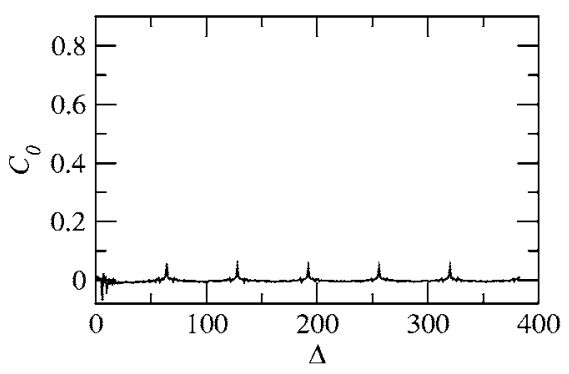

(c)



(b)



(d)



FIG. 4. The autocorrelation of attendance $C_{0}$ against various intervals $\Delta$ in (a) standard MG, and for $I=$ (b) 0.53 , (c) 0.6, and (d) 0.9 averaged over 50 independent runs. Here, $N=501, M=5$, and $S$ $=2$. 


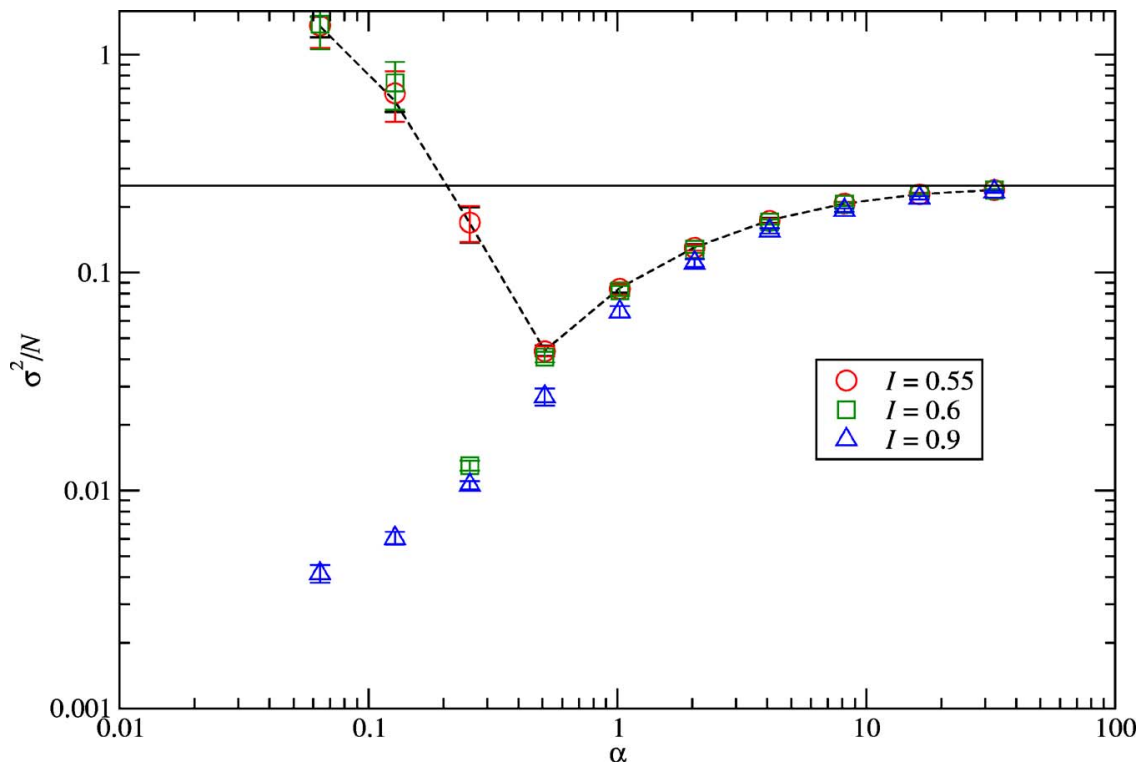

FIG. 5. (Color online) The variance of attendance per agent, $\sigma^{2} / N$, against the system complexity $\alpha$ for $I=$ (a) 0.55 , (b) 0.6 , and (c) 0.9 , with $\delta_{k}$ given by Eq. (4). Here, $N=501$ and $S=2$. The dashed line represents the $\sigma^{2} / N$ curve in the standard MG. system to escape from the grip of periodic dynamics appears to be higher. Recall that the virtual score difference of two strategies performs a random walk with the following step sizes and probabilities $p$ :

$$
\Omega_{k, 0}-\Omega_{k, 1}= \begin{cases}+2 & \text { with } p=1 / 4 \\ -2 & \text { with } p=1 / 4 \\ 0 & \text { with } p=1 / 2\end{cases}
$$

Meanwhile, the presence of periodic dynamics ensures a reset every $2^{M+1}$ time steps. We can approximately calculate the average variance by considering all possible traveling paths, which equals $2^{M+1} / 12$ (the detailed mathematics is shown in the Appendix). For instance, when $M=5$, the critical value for the periodic dynamics to disappear is $x_{c}<$ $-2 / \sqrt{2^{5+1} / 12}=-0.866$; that is, $I \simeq 0.81$. This value of $I$ is consistent with the data presented in Figs. 6 and 7. Again, we believe that after the breaking of periodic dynamics, the re- recognizing process mentioned in the previous section comes in and diversifies the strategy space, resulting in a drop of fluctuation.

\section{MIXED POPULATION WITH STANDARD MG AGENTS}

It is already clear that a pure population of agents having inertia reduces system wastage. Now it is instructive to study whether these agents (sticky agents) are advantageous over standard MG agents (sensitive agents) in a mixed population.

Figure 8 gives the success rates of both races against $\gamma$ in the mixed population with $I=0.9$, where $\gamma$ is the fraction of sticky agents in the population. Clearly, these sticky agents take advantage of the sensitive agents for the whole range of $\gamma$; they are able to maintain their success rates close to 0.5 . The sensitive agents are believed to be made more sensitive by the periodic dynamics, making them keep on losing. On the other hand, sticky agents are likely to win more fre-

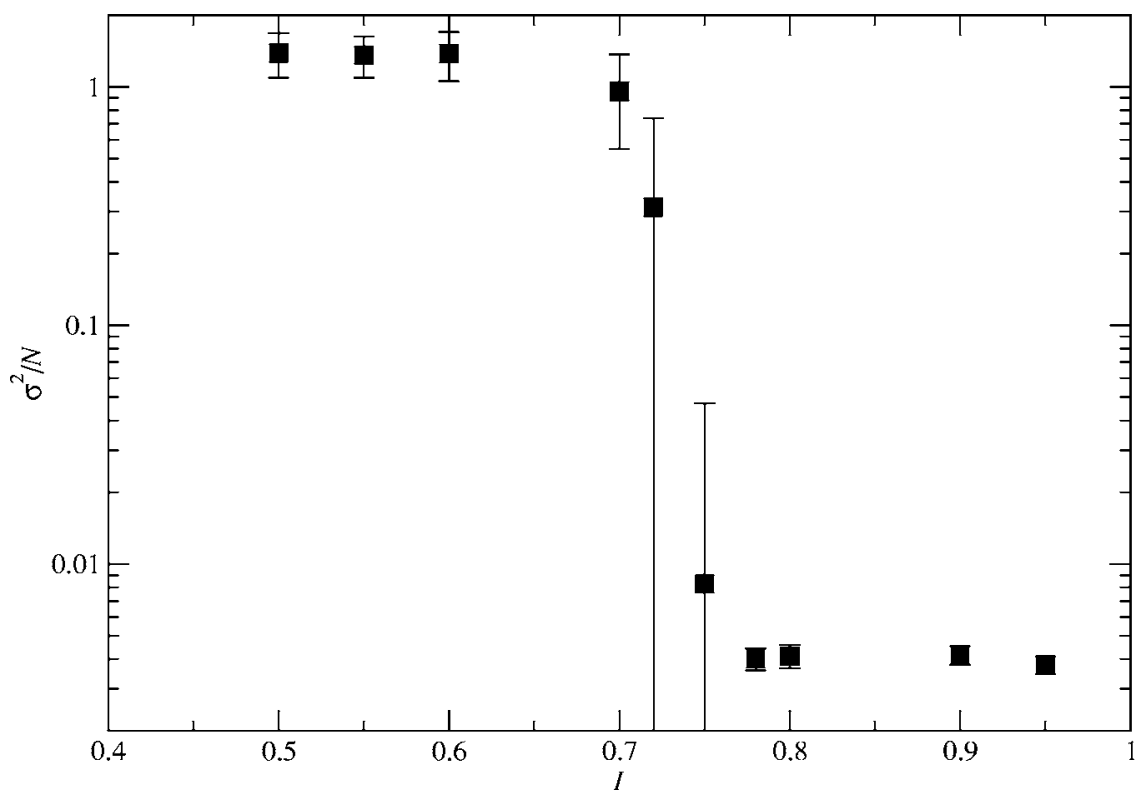

FIG. 6. The variance of attendance per agent, $\sigma^{2} / N$, against $I$ at $\alpha=0.06$, setting $\delta_{k}$ to satisfy Eq. (4). Here, $N=501, S=2$, and $M$ $=5$. 
(a)

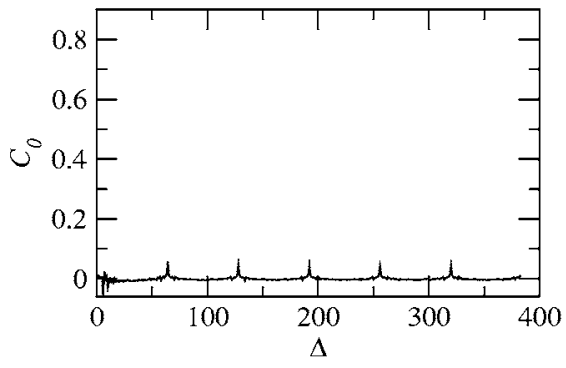

(c)

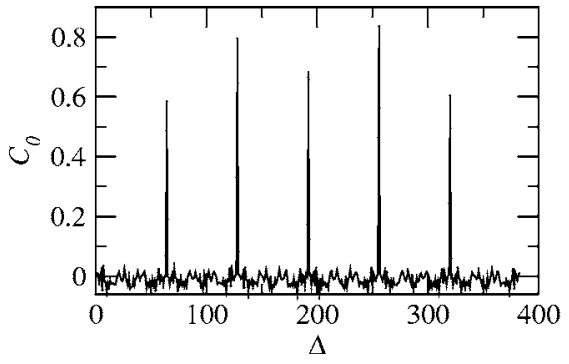

(b)

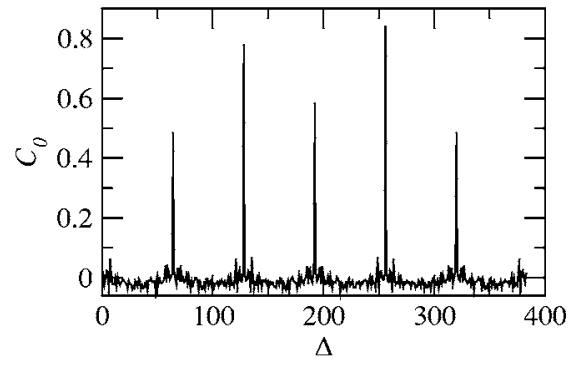

(d)

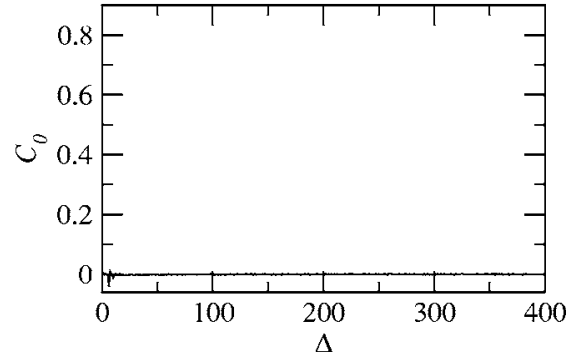

FIG. 7. The autocorrelation of attendance $C_{0}$ against various intervals $i$ on (a) standard MG, and $I=$ (b) 0.55 , (c) 0.6 , and (d) 0.9 averaged over 50 independent runs. Here, $N=501, M=5$, and $S=2$, and $\delta_{k}$ is given by Eq. (4). quently as they are resistant to following the oscillation. Note that the whole population also benefits from adding in more sticky agents (see the triangles in Fig. 8). When $\gamma$ is increased up to about $0.6, W_{s}$ starts to rise. It is because the crowd of sensitive agents is no longer large enough to override the net actions made by sticky agents, and therefore there is no more periodic dynamics existing. Figure 9 confirms our suspicion; the periodic dynamics disappears around $\gamma=0.6$. We have also performed simulations on a mixed population of sensitive agents and sticky agents with other values of $I$. As expected, sticky agents are only advantageous when $I$ exceeds the critical value that allows them to escape from the periodic dynamics mentioned in the last section. Otherwise, all agents in the whole population will still suffer from overcrowding and no one will be benefited.

\section{PREVIOUS STUDIES IN THE LITERATURE AND COMPARISON WITH OUR RESULTS}

The reduction of the volatility by modifying the rules or the initial conditions of the standard MG is not a new idea in the field, especially for the symmetric phase. A few previous studies have shown results quite similar to that of the HMG. Here, we would like to first give a short review of these works and then compare them with our study.

\section{A. Thermal minority game}

Cavagna et al. proposed the thermal minority game (TMG) [9], which adds stochasticity into the standard MG. In the TMG, an agent does not strictly employ the strategy with highest virtual score, rather he/she uses a strategy with

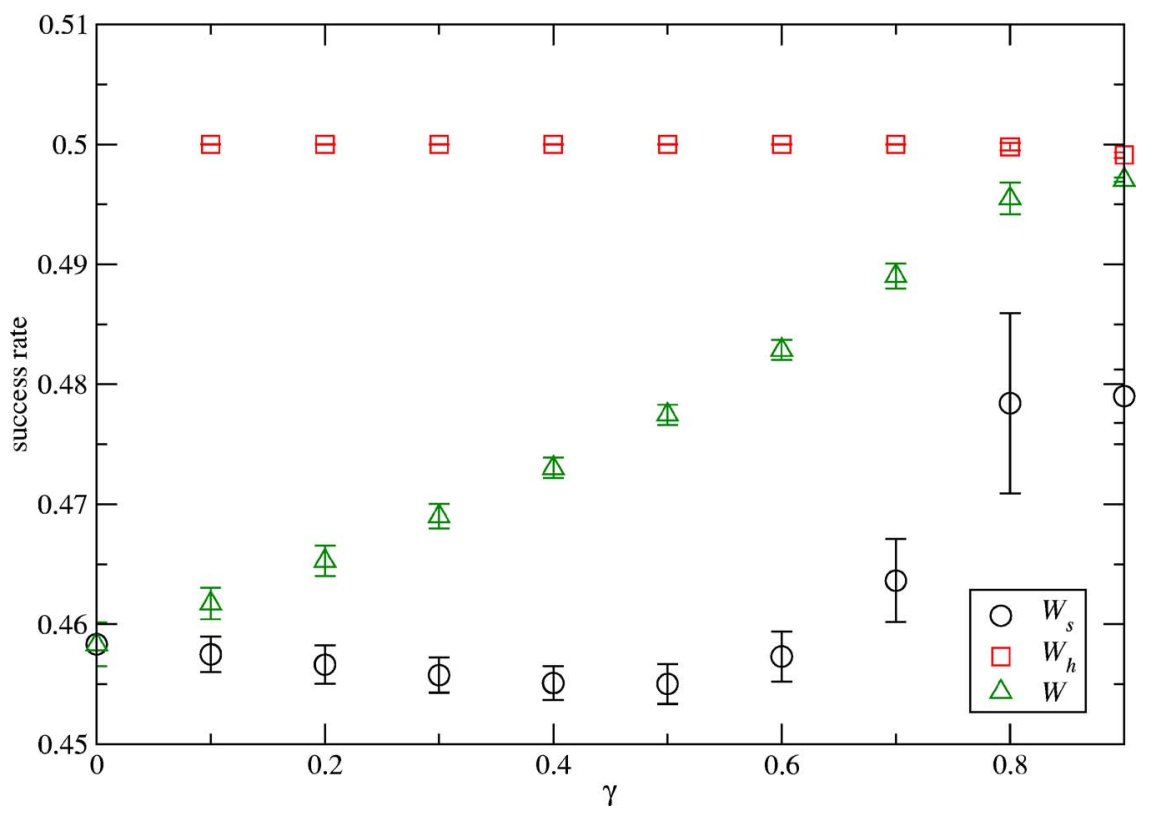

FIG. 8. (Color online) A plot of success rate of sticky agents $W_{h}$, sensitive agents $W_{s}$, and the whole population $W$ against the fraction of sticky agents $\gamma$ in the mixed group. There are total 501 agents, with $M=5, S=2$, and $I$ $=0.9$. 

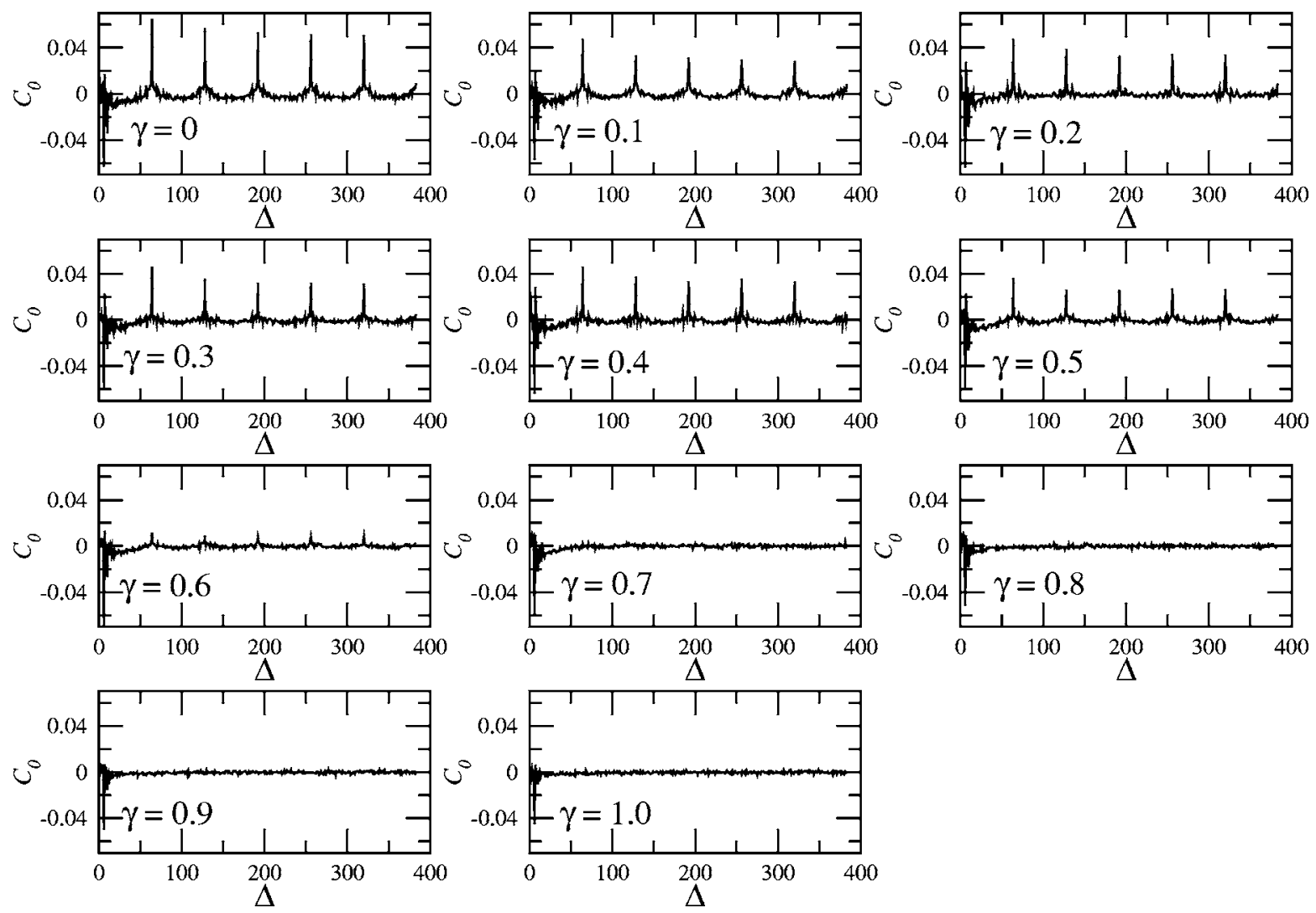

FIG. 9. The autocorrelation of attendance $C_{0}$ for different $\gamma$ averaged over 50 independent runs. Here, $N=501, M=5, S=2$, and $I=0.9$.

probability calculated according to its virtual score and a fixed inverse temperature $T$. In other words, the employment of strategies by agents become probabilistic with the degree of stochasticity depending on $T$. They found that for a certain range of $T$, the volatility in the game is reduced in most ranges of the control parameter $\alpha$. That is, in both symmetric and asymmetric phases, the TMG succeeds in raising the degree of cooperation between agents by introducing noises into the decision process of strategy selection for individual agents.

In search of the continuous time dynamics of the TMG, Garrahan et al. confirm by numerical simulation that the dynamics in the symmetric phase of the MG is sensitive to initial conditions. In particular, they reported that the volatility would drop far from the original value if random initial conditions are assigned to strategies [with $O(1)$ initial virtual scores for a population of 100 agents] at the beginning of the game [10].

\section{B. Nash equilibrium}

In searching for the replica solution and the Nash equilibrium for the symmetric phase of the standard MG, Challet et $a l$. found that the Nash equilibrium is not unique and agents at these equilibria use pure strategies (that is, they either always choose 1 or always choose 0 ) [11]. In Nash equilibrium, agents perform much better than in the standard MG; the volatility is greatly suppressed in the symmetric phase.

\section{Consideration of agents' own market impact in evaluation of strategy}

Challet et al. try to let the agents consider their own impact on the market during the evaluation of all strategies available to them. That is, the virtual score of a strategy is proportional to the cumulated payoff the agent would have received had he or she always played the strategy. Although the difference between this evaluation of virtual score and the original one is believed to be small $(\sim 1 / \sqrt{N})$, the volatility is found to be far lower than in the original MG. This difference is not negligible because of the finite size effect and the high degree of oversampling of the strategy space when $\alpha<\alpha_{c}$. However, this setting is computationally intensive and unrealistic, as a person in a real market usually can only obtain information on his/her own current wealth and is unlikely to try out all strategies [11].

\section{Analytical solutions of the batch minority game and the online minority game}

In the batch minority game, the virtual score of a particular strategy is updated as the discrete accumulated effect of order- $N$ iterations in the standard MG model, whereas the MG having the original updating method can be viewed as an "online" minority game in the neural network sense. After adding in stochasticity, initial evaluation, and generalizing these games to the continuous time limit, Coolen and coworkers have extensively written out the analytical solutions of these two versions of the MG. They found that in the 
symmetric phase their theory pointed at the existence of a critical value for the initial strategy valuations above which the system will revert to a state with vanishing volatility $[12,13]$.

\section{E. Introduction of diversity}

Wong et al. pointed out in [8] that the maladaptation observed in the symmetric phase in the standard MG originates from the fact that the initial virtual scores of all strategies are the same. They then studied the effect of introducing diversity $R / N$ into the game, where $R$ is the range of randomly assigned initial scores to strategies at the beginning of the game and $N$ is the number of agents. They found that by increasing the diversity, the maladaptive behavior observed in the symmetric phase $\alpha<\alpha_{c}$ is reduced and hence the cooperation among agents is promoted.

\section{F. Comparison to our model}

From the above studies, we can conclude that the volatility will be suppressed under the following conditions: (1) randomly allocating the initial strategy score over a critical value, (2) adding in noise or stochasticity in choosing a strategy, (3) assigning a pure strategy, or (4) taking an agent's impact on the market into account when evaluating all strategies.

First, we would like to stress that the main focus in this paper is to provide a simple formalism to incorporate inertia into a multiagent system such as the MG, as well as recording its influence on the dynamics of the game. In the HMG, there is no prior preference in strategies for they have the same initial virtual score. Unlike the standard MG, soon after the commencement of the HMG, the preference for a strategy is determined by both the virtual score differences between strategies at hand and the inertia $I_{k}$ of agent $k$. Through the presence of inertia, each agent will gradually develop its own preference in strategies through dynamical adaptation. In this respect, even though the presence of inertia may eventually lead to different views of an identical strategy between agents, this is achieved by an adaptive process through the dynamics of the system but not by artificially assigning a spread of initial virtual scores. This is a marked difference between the HMG and the work of Wong et al. [8], Garrahan et al. [10], and Heimal and Coolen $[12,13]$. More importantly, the spreading of initial virtual scores of strategies would only lead to a drop of volatility in the symmetric phase, but not the asymmetric phase. In the HMG, however, there is a global improvement in both phases for a certain value of $I$.

We believed the TMG presents results most similar to our game. In both cases, the degree of cooperation is increased in most ranges of $\alpha$. However, as mentioned previously, the TMG achieves this by adding stochasticity and noise into the agents' choice of best strategies. Meanwhile, in the HMG the agents are deterministic when choosing their best strategies: they stick to their current strategy until it is outperformed at a certain threshold; this does not involve any stochasticity. In fact, the dynamics of the HMG is deterministic when played by an odd number of agents each carrying two strategies.
Lastly, we think that using pure strategies and taking agents themselves into account when evaluating all their strategies is impractical and an unrealistic situation. Our model provides a natural, realistic way to prompt cooperation, meanwhile demonstrating the effect of stickiness when people change their investment strategies.

\section{CONCLUSIONS}

We have successfully introduced the concept of inertia into the minority game, which shows a remarkable improvement of cooperation among agents in most ranges of $\alpha$, especially in the symmetric phase $\alpha<\alpha_{c}$. We also compare our findings with a few variants of the MG reported in the literature. We calculated the critical values of inertia needed to uplift the cooperation behaviors, which depends on how agents perceive the fluctuation of the virtual score difference between strategies. This reduction of sensitivity among agents is found to be useful in removing maladaptation due to over-reaction. In contrast, if every action is smooth and all agents respond to information in no time, they will suffer from overcrowding easily. Meanwhile, agents carrying stickiness seem to perform much better than sensitive agents. Our findings suggest that inertia (or stickiness) is crucial and beneficial to a society. It is hoped that the role of inertia will be investigated in more detail based on our model HMG; the effect of giving a diversifying range of inertia to a population would be interesting. It is also instructive to apply our method of modeling inertia to study the inertia effect in other multiagent systems.

\section{ACKNOWLEDGMENTS}

We thank the Computer Center of HKU for their helpful support in providing the use of the HPCPOWER system for simulations reported in this paper. K. M. Lee, F. K. Chow, K. H. Ho, and C. C. Leung are gratefully acknowledged for their fruitful discussions.

\section{APPENDIX}

In this appendix, we consider a simple random walk of cumulative sum $x_{t}$ after time $t$. At each step, $x_{t}$ increases (decreases) by 1 if the article moves upward (downward) with probability $1 / 2$. We also impose a boundary condition that the sum is equal to 0 at both $t=0$ and $t=T$. A schematic diagram is shown in Fig. 10. Under this constraint, we find-

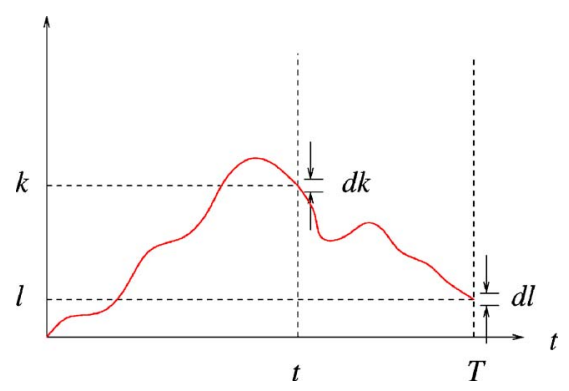

FIG. 10. (Color online) A schematic sketch showing a typical random walk of a particle traveling for $T$ time step. 
that the variance of $x_{t}$ averages over all possible paths $\sigma_{r}^{2}$ $=t(T-t) / 4 T$. Using this formula, we can evaluate the average standard deviation of the virtual score difference of an agent's strategies within a period $2^{M+1}$, that is, the $\delta_{k}$ mentioned in Eq. (4).

First, we need to know the probability of $x_{t}$ within $k$ and $k+d k$, which is given by [27]

$$
P\left(k \leqslant x_{t} \leqslant k+d k\right) \approx \sqrt{\frac{2}{\pi t}} e^{-2 k^{2} / t} d k
$$

Hence, the probability of the cumulative sum $x_{t}$ to be within $k$ and $k+d k$ at time $t$ and $x_{T}$ to be within $l$ and $l+d l$ at time $T$ can be expressed by

$$
\begin{aligned}
P(k & \left.\leqslant x_{t} \leqslant k+d k \text { and } l \leqslant x_{T} \leqslant l+d l\right) \\
& =P\left(k \leqslant x_{t} \leqslant k+d k\right) P\left[l-k \leqslant x_{T-t} \leqslant l-k+d l\right]
\end{aligned}
$$

where $t \leqslant T$. The equality follows from the fact that the discrete step size is equal to 1. Using Eqs. (A1) and (A2), the conditional probability

$$
\begin{aligned}
P(k & \left.\leqslant x_{t} \leqslant k+d k \mid l \leqslant x_{T} \leqslant l+d l\right) \\
& =\frac{P\left(k \leqslant x_{t} \leqslant k+d k \text { and } l \leqslant x_{T} \leqslant l+d l\right)}{P\left(l \leqslant x_{T} \leqslant l+d l\right)}
\end{aligned}
$$

$$
=\frac{\sqrt{2 / \pi t} e^{-2 k^{2} / t} \sqrt{2 / \pi(T-t)} e^{-2(l-k)^{2} /(T-t)} d k d l}{\sqrt{2 / \pi T} e^{-2 l^{2} / T} d l} .
$$

By the boundary condition $0 \leqslant x_{T} \leqslant d l$, then we have

$$
P\left(k \leqslant x_{t} \leqslant k+d k \mid 0 \leqslant x_{T} \leqslant d l\right)=\sqrt{\frac{2 T}{\pi t(T-t)}} e^{-2 T k^{2} / t(T-t)} d k .
$$

Therefore, the variance $\sigma_{r}^{2}$ averaged over all possible paths is

$$
\begin{aligned}
\sigma_{r}^{2} & =\int_{-\infty}^{+\infty} k^{2} P\left(k \leqslant x_{t} \leqslant k+d k \mid 0 \leqslant x_{T} \leqslant d l\right) \\
& =\frac{t(T-t)}{4 T} .
\end{aligned}
$$

In order to calculate $\delta_{k}^{2}$, we should rescale $\sigma_{r}^{2}$ because the virtual score difference of an agent's strategies can move two steps upward $(+2)$ or two steps downward $(-2)$ or stay stationary (0). Hence, by approximating the travel time $T$ as consisting of an infinit number of time steps:

$$
\delta_{k}^{2}=2\left(\frac{1}{T}\right) \int_{0}^{T} \frac{t(T-t)}{4 T} d t=\frac{T}{12} .
$$

where $\delta_{k}$ is the perceived fluctuation mentioned in Eq. (4).
[1] S. A. Levin, Ecosystems 1, 431 (1998).

[2] P. Laureti and Y. C. Zhang, Physica A 324, 49 (2003).

[3] A. Chatterjee, B. L. Chakrabarti, and S. S. Manna, Physica A 335, 155 (2004).

[4] W. B. Arthur, Am. Econ. Rev. 84, 406 (1994).

[5] D. Challet and Y. C. Zhang, Physica A 246, 407 (1997).

[6] D. Kahneman and A. Tversky, Econometrica 47, 263 (1979).

[7] A. Tversky and D Kahneman, J. Risk and Uncertainty 5, 297 (1992).

[8] K. Y. Michael Wong, S. W. Lim, and Z. Gao, Phys. Rev. E 70, 025103(R) (2004).

[9] A. Cavagna, J. P. Garrahan, I. Giardina, and D. Sherrington, Phys. Rev. Lett. 83, 108 (1999).

[10] J. P. Garragan, E. Moro, and D. Sherrington, Phys. Rev. E 62, R1 (2000).

[11] D. Challet, M. Marsili, and R. Zecchina, Phys. Rev. Lett. 84, 1824 (2000).

[12] J. A. F. Heimel and A. C. C. Coolen, Phys. Rev. E 63, 056121 (2001).

[13] A. C. C. Coolen and J. A. F. Heimel, J. Phys. A 34, 10783 (2001).

[14] Y. C. Zhang, Europhys. News 29, 51 (1998).

[15] N. F. Johnson, P. Jefferies, and P. M. Hui, Financial Market
Complexity: What Physics Can Tell Us about Market Behaviour (Oxford University Press, Oxford, 2003).

[16] D. Challet, M. Marsili, and Y.-C. Zhang, Minority Games (Oxford University Press, Oxford, 2005).

[17] A. C. C. Coolen, The Mathematical Theory Of Minority Games: Statistical Mechanics Of Interacting Agents (Oxford University Press, Oxford, 2005).

[18] D. Challet and Y. C. Zhang, Physica A 256, 514 (1998).

[19] R. Manuca, Y. Li, R. Riolo, and R. Savit, Physica A 282, 559 (2000).

[20] R. Savit, R. Manuca, and R. Riolo, Phys. Rev. Lett. 82, 2203 (1999).

[21] D. Challet and M. Marsili, Phys. Rev. E 60, R6271 (1999).

[22] M. Hart, P. Jefferies, N. F. Johnson, and P. M. Hui, Physica A 298, 537 (2001).

[23] M. Hart, P. Jefferies, N. F. Johnson, and P. M. Hui, Eur. Phys. J. B 20, 547 (2001)

[24] D. Challet and M. Marsili, Phys. Rev. E 62, 1862 (2000).

[25] C. Y. Lee, Phys. Rev. E 64, 015102(R) (2001).

[26] K. H. Ho, W. C. Man, F. K. Chow, and H. F. Chau, Phys. Rev. E 71, 066120 (2005).

[27] A. N. Shiryaev, Probability, 2nd ed. (Springer, Berlin, 1989), p. 49. 